\title{
Origin of the pressure-dependent Tc valley in superconducting simple cubic phosphorus
}

\author{
Wu, Xianxin ; Jeschke, Harald O ; Di Sante, Domenico ; von Rohr, Fabian O ; Cava, Robert J ;
} Thomale, Ronny

\begin{abstract}
Motivated by recent experiments, we investigate the pressure-dependent electronic structure and electron-phonon (e-ph) coupling for simple cubic phosphorus by performing first-principles calculations within the full potential linearized augmented plane-wave method. As a function of increasing pressure, our calculations show a valley feature in Tc, followed by an eventual decrease for higher pressures. We demonstrate that this Tc valley at low pressures is due to two nearby Lifshitz transitions, as we analyze the band-resolved contributions to the e-ph coupling. Below the first Lifshitz transition, the phonon hardening and shrinking of the Fermi surface with s-orbital character results in a decreased Tc with increasing pressure. After the second Lifshitz transition, the appearance of Fermi surfaces with 3d-orbital character generate strong e-ph interband couplings in and channels, and hence lead to an increase of Tc. For higher pressures, the phonon hardening finally dominates, and Tc decreases again. Our study reveals that the intriguing Tc valley discovered in experiment can be attributed to Lifshitz transitions, while the plateau of Tc detected at intermediate pressures appears to be beyond the scope of our analysis. This strongly suggests that aside from e-ph coupling, electronic correlations along with plasmonic contributions may be relevant for simple cubic phosphorus. Our findings hint at the notion that increasing pressure can shift the low-energy orbital weight towards d character, and as such even trigger an enhanced importance of orbital-selective electronic correlations despite an increase of the overall bandwidth.
\end{abstract}

DOI: https://doi.org/10.1103/physrevmaterials.2.034802

Posted at the Zurich Open Repository and Archive, University of Zurich

ZORA URL: https://doi.org/10.5167/uzh-167498

Journal Article

Published Version

Originally published at:

Wu, Xianxin; Jeschke, Harald O; Di Sante, Domenico; von Rohr, Fabian O; Cava, Robert J; Thomale, Ronny (2018). Origin of the pressure-dependent Tc valley in superconducting simple cubic phosphorus. Physical Review Materials, 2(3):034802.

DOI: https://doi.org/10.1103/physrevmaterials.2.034802 


\title{
Origin of the pressure-dependent $T_{c}$ valley in superconducting simple cubic phosphorus
}

\author{
Xianxin Wu, ${ }^{1, *}$ Harald O. Jeschke,,${ }^{1,2}$ Domenico Di Sante, ${ }^{1}$ Fabian O. von Rohr,,${ }^{3,4}$ Robert J. Cava, ${ }^{3}$ and Ronny Thomale ${ }^{1}$ \\ ${ }^{1}$ Institut für Theoretische Physik und Astrophysik, Julius-Maximilians-Universität Würzburg, 97074 Würzburg, Germany \\ ${ }^{2}$ Research Institute for Interdisciplinary Science, Okayama University, Okayama 700-8530, Japan \\ ${ }^{3}$ Department of Chemistry, Princeton University, Princeton, New Jersey 08544, USA \\ ${ }^{4}$ Department of Chemistry, University of Zurich, $\mathrm{CH}-8057$, Switzerland
}

(Received 19 December 2017; published 27 March 2018)

\begin{abstract}
Motivated by recent experiments, we investigate the pressure-dependent electronic structure and electronphonon (e-ph) coupling for simple cubic phosphorus by performing first-principles calculations within the full potential linearized augmented plane-wave method. As a function of increasing pressure, our calculations show a valley feature in $T_{c}$, followed by an eventual decrease for higher pressures. We demonstrate that this $T_{c}$ valley at low pressures is due to two nearby Lifshitz transitions, as we analyze the band-resolved contributions to the e-ph coupling. Below the first Lifshitz transition, the phonon hardening and shrinking of the $\gamma$ Fermi surface with $s$-orbital character results in a decreased $T_{c}$ with increasing pressure. After the second Lifshitz transition, the appearance of $\delta$ Fermi surfaces with $3 d$-orbital character generate strong e-ph interband couplings in $\alpha \delta$ and $\beta \delta$ channels, and hence lead to an increase of $T_{c}$. For higher pressures, the phonon hardening finally dominates, and $T_{c}$ decreases again. Our study reveals that the intriguing $T_{c}$ valley discovered in experiment can be attributed to Lifshitz transitions, while the plateau of $T_{c}$ detected at intermediate pressures appears to be beyond the scope of our analysis. This strongly suggests that aside from e-ph coupling, electronic correlations along with plasmonic contributions may be relevant for simple cubic phosphorus. Our findings hint at the notion that increasing pressure can shift the low-energy orbital weight towards $d$ character, and as such even trigger an enhanced importance of orbital-selective electronic correlations despite an increase of the overall bandwidth.
\end{abstract}

DOI: 10.1103/PhysRevMaterials.2.034802

\section{INTRODUCTION}

Layered black phosphorus (black P), a narrow-gap semiconductor, crystallizes in the orthorhombic $A 17$ structure at ambient pressure and temperature, with layers weakly bound by van der Waals forces [1,2]. The monolayer phosphorene is similar to graphene, albeit with a natural band gap [3-5]. Recently, topologically nontrivial semimetallic properties have been discovered in the $A 17$ structure at low pressure (i.e., a few GPa) [6-9], which contributed to revitalizing the interest in this material. Under pressure, early studies showed that black phosphorus exhibits several phase transitions $[1,2]$. The structure changes from $A 17$ (orthorhombic) to semimetallic $A 7$ (rhombohedral) at $4.5 \mathrm{GPa}$, followed by a metallic primitive cubic phase at $10 \mathrm{GPa}[1,2]$. The former transition is sluggish while the latter is rather sharp. It is remarkable to note that although the atoms are not closely packed [10], the primitive cubic (or simple cubic) phase is stable up to a pressure of $107 \mathrm{GPa}$ before it starts to transform into a hexagonal phase, completed at $137 \mathrm{GPa}$ [11]. Aside from the unusually rich variety of structural phase transitions, superconductivity was discovered in the rhombohedral and cubic phases above $5 \mathrm{GPa}$ [12-14]. Put together, this naturally suggests black $\mathrm{P}$ to be a prime candidate for a particularly interesting shape of $T_{c}$ as a function of different trajectories taken with respect to pressure and temperature. Accordingly, the pressure dependence of

\footnotetext{
*Xianxin.Wu@physik.uni-wuerzburg.de
}

$T_{c}$ has been investigated by many groups. Still, there is no experimental consensus on this question. This is because (i) the measured $T_{c}$ appears to be strongly dependent on the path taken in $P-T$ parameter space $[13,14]$ and (ii), probably not uncorrelated with (i), there are mixed states due to incomplete structural phase transformations. Kawamura et al. [13,14] found that $T_{c}$ increased continuously with pressure in the cubic phase on one path, while it varied only weakly with pressure around $6 \mathrm{~K}$ on the other path. Wittig et al. [12] discovered that $T_{c}$ showed two distinct peaks at about 12 and $23 \mathrm{GPa}$ separated by an intriguing valley centered at about $17 \mathrm{GPa}$. In contrast, Karuzawa et al. [15] showed that $T_{c}$ exhibits only a single peak around $23 \mathrm{GPa}$, and the corresponding $T_{c}$ was about $9.5 \mathrm{~K}$. In the most recent experiment, performed with high-quality black P crystals and excellent pressure cell conditions, Guo et al. [16] found a behavior similar to that of Wittig et al. in terms of pressure variation of $T_{c}$ : in the cubic phase, with increasing pressure, $T_{c}$ first decreases and then increases, i.e., it forms a $T_{c}$ valley. From about $32 \mathrm{GPa}$, $T_{c}$ exhibits a plateau for an extended pressure regime at a comparably high value. Moreover, Hall effect measurements suggested that hole carriers play an important role in promoting superconductivity, and that the $T_{c}$ valley may originate from a Lifshitz transition [16].

From an initial theoretical point of view, black $\mathrm{P}$ does not promise to be particularly controversial. As the $T_{c}$ for cubic phosphorus is relatively low (with a maximum of about $10 \mathrm{~K}$ ) and phosphorus has an approximately half-filled $p$ band, superconductivity has so far been assumed to be driven 
by electron phonon (e-ph) coupling. No consensus, however, has been reached on the pressure dependence of $T_{c}$ in the cubic phase, as the result strongly depends on the adopted theoretical methods. By relating average phonon frequency and bulk modulus, early calculations [17] show a single peaked structure for $T_{c}$ as a function of pressure, where the peak position is nearby the second peak of the experimental data in Ref. [12]. It must be noted, however, that the variation of the phonon spectrum under pressure is completely omitted in this analysis. Other calculations have mainly focused on the electronic structure [18], or find that $T_{c}$ changes little with increasing pressure [19], which is only consistent with the data on one particular path through $P-T$ space [13]; it is inconsistent with recent experimental evidence. Another study finds that $T_{c}$ increases slightly from 8.5 to $11 \mathrm{~K}$ as the pressure increases from 10 to $35 \mathrm{GPa}$ [20], which again hardly bears similarity to experiments. A more recent, and technically significantly advanced, study involving full $a b$ initio calculations of e-ph coupling using pseudopotentials finds that $T_{c}$ decreases monotonously in the simple cubic phase [21], and reports no indication for any valley or plateau formation. Furthermore, in the latest theoretical studies, metastable structures in black P are found to be important to explain the $P-T$ path dependent $T_{c}$ in experiments [22].

In this paper, we pursue two objectives. First, facing partly contradictory experimental evidence, we attempt to consider all experimental facts on the same footing, but if in doubt center our experimental reference around Ref. [16], and apply $a b$ initio methods to the most refined level in order to provide a theoretical explanation. In particular, as justified by the crystal analysis performed in Ref. [16], we explicitly start from the hypothesis that the structural transition to the simple cubic phase sets in comparably early, and that the $T_{c}$ valley, as observed, occurs within this phase. By employing more reliable full potential calculations instead of pseudopotential calculations, we attempt to resolve previous discrepancies in theoretical calculations. We manage to explain the $T_{c}$ valley formation found experimentally, as the precision of our analysis allows us to disentangle effects of Lifshitz transitions from structural transitions. Second, we try to sharpen the perspective on how the $a b$ initio analysis would have to be extended in order to refine the correspondence between theory and experiment. As we investigate electronic correlations in simple cubic black P, we notice several interesting aspects that might be relevant for a larger class of materials under pressure. For instance, we find that the $d$-orbital low-energy weight increases as a function of pressure. In total, we are led to conclude that orbital-selective interactions as well as plasmonic contributions might provide central additional insights into the persistent intricacies of black $\mathrm{P}$ under pressure.

The paper is organized as follows. In Sec. II, as also supplemented by the Appendices, we introduce the detailed formalism of our $a b$ initio analysis for the electronic and crystal structure as well as the e-ph coupling. For the former, as opposed to previous studies, we employ full potential calculations. For the latter, we likewise refine previous studies by considering band-selective e-ph coupling strengths. Section III proceeds by giving a detailed account on the results of our analysis for simple cubic black $P$. This also includes a detailed orbital-resolved study of the electronic structure, as well as the calculation of e-ph coupling via different $a b$ initio approaches. As we are bringing together our theoretical findings with the experimental evidence in Sec. IV, we can quantitatively and qualitatively rationalize the $T_{c}$ valley formation in the simple cubic phase. In Sec. V, we conclude that while we are still short of an answer to some of the experimental features, in particular to those observed for higher pressures, our systematic study supports our resulting hypothesis that electronic $a b$ initio approaches are insufficient to cover the full phenomenology of black P. Instead, we suggest that electronic correlations may enter within a degree of sophistication that is beyond such methods, and that plasmonic contributions are likely to improve the current theoretical understanding of the material.

\section{METHODS}

\section{A. Electron-phonon coupling formalism}

We start by describing the e-ph coupling formalism we employ. The e-ph matrix element for the scattering of an electron in band $n$ at wave vector $\mathbf{k}$ to a state in band $m$ with wave vector $\mathbf{k}+\mathbf{q}$, with the absorption or emission of a phonon with mode $v$ at wave vector $\mathbf{q}$, is

$$
g_{m n}^{\nu}(\mathbf{k}, \mathbf{q})=\sqrt{\frac{\hbar}{2 M \omega_{\mathbf{q} v}}}\left\langle m, \mathbf{k}+\mathbf{q}\left|\delta_{\mathbf{q} \nu} V_{\mathrm{SCF}}\right| n, \mathbf{k}\right\rangle .
$$

In (1), $|n, \mathbf{k}\rangle$ is the electronic Bloch state, $\omega_{\mathbf{q} v}$ is the screened phonon frequency, $M$ is the atomic mass, and $\delta_{\mathbf{q} v} V_{\mathrm{SCF}}$ is the derivative of the self-consistent potential with respect to the collective atomic displacements corresponding to phonon mode $v$ at wave vector $\mathbf{q}$. The phonon self-energy is given by

$$
\Pi_{\mathbf{q} v}=\frac{1}{N_{k}} \sum_{m n, \mathbf{k}}\left|g_{m n}^{v}(\mathbf{k}, \mathbf{q})\right|^{2} \frac{n_{\mathrm{F}}\left(\epsilon_{m \mathbf{k}+\mathbf{q}}\right)-n_{\mathrm{F}}\left(\epsilon_{n \mathbf{k}}\right)}{\omega_{\mathbf{q} \nu}+i \delta+\epsilon_{m \mathbf{k}+\mathbf{q}}-\epsilon_{n \mathbf{k}}}
$$

where $\epsilon_{n \mathbf{k}}$ is the energy relative to the Fermi level for the Bloch state, $n_{\mathrm{F}}(\epsilon)$ is the Fermi distribution function, and $N_{k}$ is the number of $k$ points. The phonon linewidth is proportional to the imaginary part of the phonon self-energy. Considering the large value of the Fermi energy compared to the phonon frequencies, the phonon linewidth at low temperature can be written as [23]

$$
\gamma_{\mathbf{q} v}(\omega)=\frac{2 \pi \omega_{\mathbf{q} v}}{N_{k}} \sum_{m n, \mathbf{k}}\left|g_{m n}^{\nu}(\mathbf{k}, \mathbf{q})\right|^{2} \delta\left(\epsilon_{m \mathbf{k}+\mathbf{q}}\right) \delta\left(\epsilon_{n \mathbf{k}}\right) .
$$

The e-ph coupling constant for a specific phonon mode is

$$
\lambda_{\mathbf{q} v}=\frac{\gamma_{\mathbf{q} v}}{\pi N\left(E_{\mathrm{F}}\right) \omega_{\mathbf{q} v}^{2}},
$$

where $N\left(E_{\mathrm{F}}\right)$ is the density of states per spin at the Fermi level. In terms of the phonon linewidths, the Eliashberg spectral function $\alpha^{2} F(\omega)$ can be written as

$$
\alpha^{2} F(\omega)=\frac{1}{2 \pi N\left(E_{\mathrm{F}}\right)} \sum_{\mathbf{q} v} \frac{\gamma_{\mathbf{q} v}}{\omega_{\mathbf{q} v}} \delta\left(\omega-\omega_{\mathbf{q} v}\right) .
$$

Finally, the isotropic e-ph coupling constant is defined as

$$
\lambda=2 \int_{0}^{\infty} d \omega \frac{\alpha^{2} F(\omega)}{\omega} .
$$

For multiband systems, as is the case for simple cubic $\mathrm{P}$, it is useful to introduce a band-resolved e-ph coupling constant 
$c_{m n}$, which describes the Cooper pair scattering of an electron from band $m$ to band $n$ by phonons, and is defined as [24]

$$
\begin{aligned}
c_{m n} & =V_{m n} N_{m} N_{n} \\
& =\frac{2}{N_{\boldsymbol{q}} N_{\boldsymbol{k}}} \sum_{\boldsymbol{k} \in n, \boldsymbol{k}+\boldsymbol{q} \in m, v} \omega_{\mathbf{q} v}^{-1}\left|g_{m n}^{\nu}(\mathbf{k}, \mathbf{q})\right|^{2} \delta\left(\epsilon_{m \mathbf{k}+\mathbf{q}}\right) \delta\left(\epsilon_{n \mathbf{k}}\right),
\end{aligned}
$$

where $N_{m}\left(N_{n}\right)$ is the density of states (DOS) at the Fermi level contributed by $m$ th ( $n$ th) band and $V_{m n}$ is the effective interaction for Cooper pair scattering. The isotropic e-ph coupling constant can be written as

$$
\lambda=\sum_{m n} \frac{c_{m n}}{N\left(E_{F}\right)}=\sum_{m n} \frac{V_{m n} N_{m} N_{n}}{N\left(E_{F}\right)} .
$$

In multiband systems, the e-ph constant is given by the maximum eigenvalue $\lambda_{\text {multi }}$ of the matrix $\Lambda$, which is defined as

$$
\Lambda_{m n}=V_{m n} N_{n}=\frac{c_{m n}}{N_{m}} .
$$

The band-resolved Eliashberg function $\alpha^{2} F_{m n}(\omega)$ reads as

$$
\begin{aligned}
& \alpha^{2} F_{m n}(\omega) \\
& \quad=\sum_{\boldsymbol{k} \in n, \boldsymbol{k}+\boldsymbol{q} \in m, v} \frac{\left|g_{m n}^{\nu}(\mathbf{k}, \mathbf{q})\right|^{2} \delta\left(\epsilon_{m \mathbf{k}+\mathbf{q}}\right) \delta\left(\epsilon_{n \mathbf{k}}\right) \delta\left(\omega-\omega_{\mathbf{q} v}\right)}{N_{n} N_{\boldsymbol{q}} N_{\boldsymbol{k}}} .
\end{aligned}
$$

Then, the matrix element $\Lambda_{m n}$ can be written as

$$
\Lambda_{m n}=2 \int_{0}^{\infty} d \omega \frac{\alpha^{2} F_{m n}(\omega)}{\omega} .
$$

$T_{c}$ can be determined using the Allen-Dynes-modified McMillan equation $[25,26]$

$$
T_{\mathrm{c}}=\frac{\omega_{l n}}{1.2} \exp \left\{-\frac{1.04(1+\lambda)}{\lambda-\mu^{*}(1+0.62 \lambda)}\right\},
$$

where $\mu^{*}$ is the Coulomb pseudopotential [27] and $\lambda$ is the isotropic (or multiband) e-ph coupling constant. $\omega_{l n}$ is defined as

$$
\omega_{l n}=\exp \left\{\frac{2}{\lambda} \int_{0}^{\infty} d \omega \ln \omega \frac{\alpha^{2} F(\omega)}{\omega}\right\} .
$$

Using Eq. (12), we obtain $T_{c}$ based on a suitable choice of $\mu^{*}$ and compare with previous works. However, we also use the solution of the isotropic Eliashberg equations to determine $T_{c}$ without any empirical parameters [28,29].

\section{B. Details of the calculations}

In the relaxation with external pressure, we employ the projector augmented wave (PAW) method [30] as implemented in the Vienna ab initio simulation package (VASP) [31-33], where both the local density approximation (LDA) and the generalized-gradient approximation (GGA) [34] for the exchange-correlation functional are used. The expansion of the wave functions in the plane-wave basis is cut off at $600 \mathrm{eV}$. The adopted $k$-point mesh is $37 \times 37 \times 37$ and the convergence criterion is that all the forces on atoms are less than $0.001 \mathrm{eV} / \AA$. The e-ph coupling calculations are performed with the full potential linear augmented plane-wave (FPLAPW) method [35] as implemented in the ELK code [36,37].
Note that by choosing all electron calculations, we ensure high accuracy of the electronic structure even under high pressures. In the calculations, we chose the Perdew-Burke-Ernzerhof (PBE) exchange-correlation functional. The adopted muffintin radii $R_{\mathrm{MT}}$ (2.05-2.20 a.u.) for $\mathrm{P}$ depend on the lattice constants and the plane-wave cutoff is set to $R_{\mathrm{MT}} \times k_{\max }=8.5$. The angular momentum expansion in the muffin tins is taken to $l_{\max }=12$ for both the wave functions and the potential. We use the full potential local orbital (FPLO) basis [38] as a further accuracy check for the electronic structure. For accurate DOS calculations, we use $\boldsymbol{k}$-point meshes of $100 \times 100 \times 100$. Phonon dispersions are obtained by using the supercell method, and we have carefully checked the convergence of the phonon spectrum with respect to $q$-point mesh. We find that an $8 \times 8 \times 8 q$-point mesh is enough to obtain the converged phonon spectrum. For the phonon linewidth in Eq. (3), in ELK the $\delta$ function is numerically replaced by a Gaussian function with a smearing parameter $\sigma$. The convergence of $\gamma_{v q}$ with respect to $k$-point mesh has also been carefully checked, and the final results are obtained from calculations with a $128 \times 128 \times 128 k$-point mesh and $\sigma=0.004 \mathrm{Ha} \approx$ $100 \mathrm{meV}$. With the chosen parameters, $\lambda$ is estimated to converge within 0.01 (i.e., less than $2 \%$ ). We also perform e-ph calculations to obtain the resistivity by using the EPW (short for electron-phonon coupling using Wannier functions) method [39,40], as implemented in the QUANTUM ESPRESSO pseudopotential plane-wave code package [41].

\section{RESULTS}

\section{A. Crystal and electronic structure for simple cubic phosphorus}

The calculated lattice constants as a function of external pressure for simple cubic phosphorus are shown in Fig. 1, in comparison with experimental data [16,42]. The lattice constants from PBE are always slightly larger than those of LDA. From the figure, we find that the calculated lattice constants from PBE match better with available experimental data, suggesting that the PBE functional may be more suitable for $\mathrm{P}$ than the LDA functional. Therefore, we adopted the

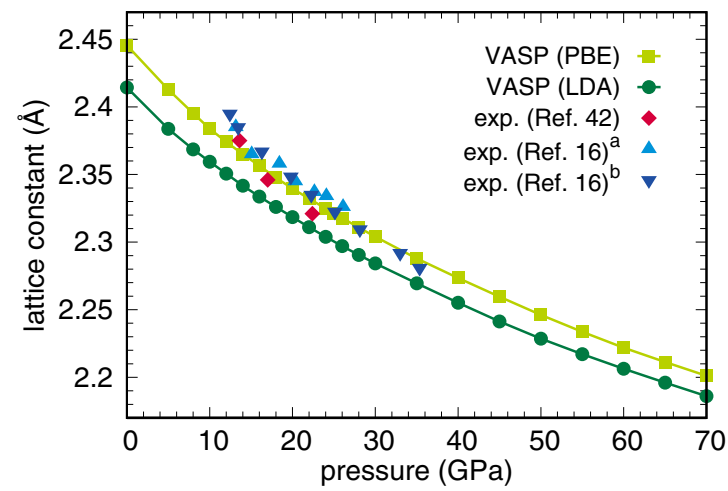

FIG. 1. Theoretical and experimental lattice constants as a function of external pressure for simple cubic phosphorus. Calculations are performed with VASP using two different exchange-correlation functionals, and experimental data are taken from Refs. [16,42] (superscripts a and b refer to two different runs). 

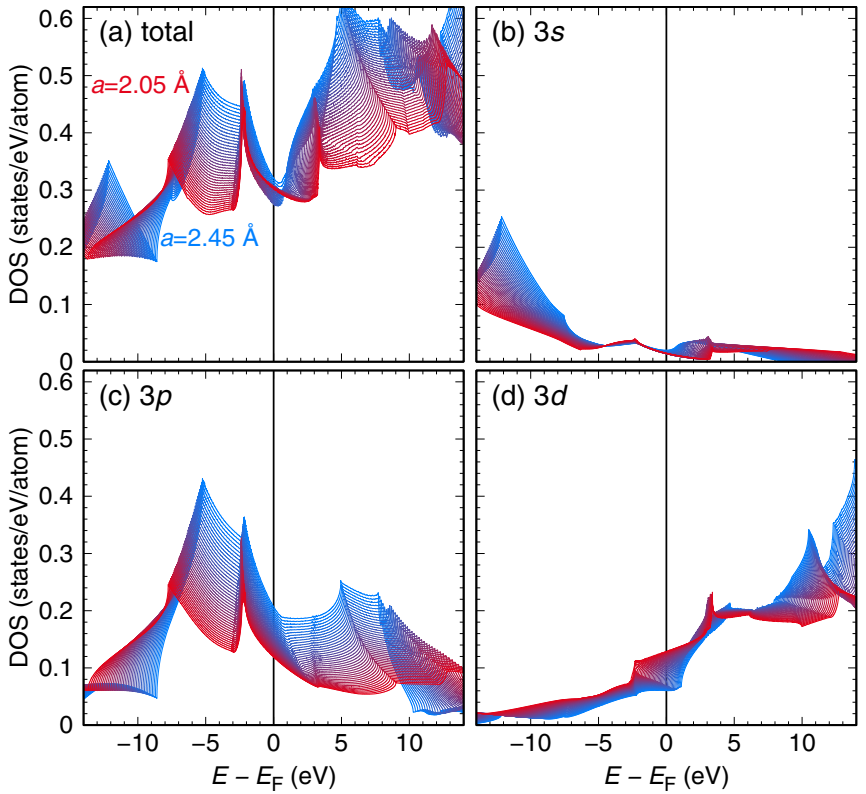

FIG. 2. Total and orbital projected densities of states in the simple cubic phase for various lattice constants. This calculation was done with FPLO. The gradient lines ranging from blue to red represent the increasing pressures (decreasing lattice constants).

PBE functional in all further calculations. The total density of states (DOS) and the orbital-projected DOS are shown in Fig. 2. At ambient pressure, the states near the Fermi level $E_{\mathrm{F}}$ are mainly formed by $p$ orbitals. As the pressure increases, however, the total states display shifts to lower energy, and the contribution from $p$-and $s$-orbital states at $E_{\mathrm{F}}$ decreases while the $d$-orbital weight increases. The DOS at the Fermi level $N\left(E_{\mathrm{F}}\right)$ as a function of external pressure is shown in Fig. 3(a).

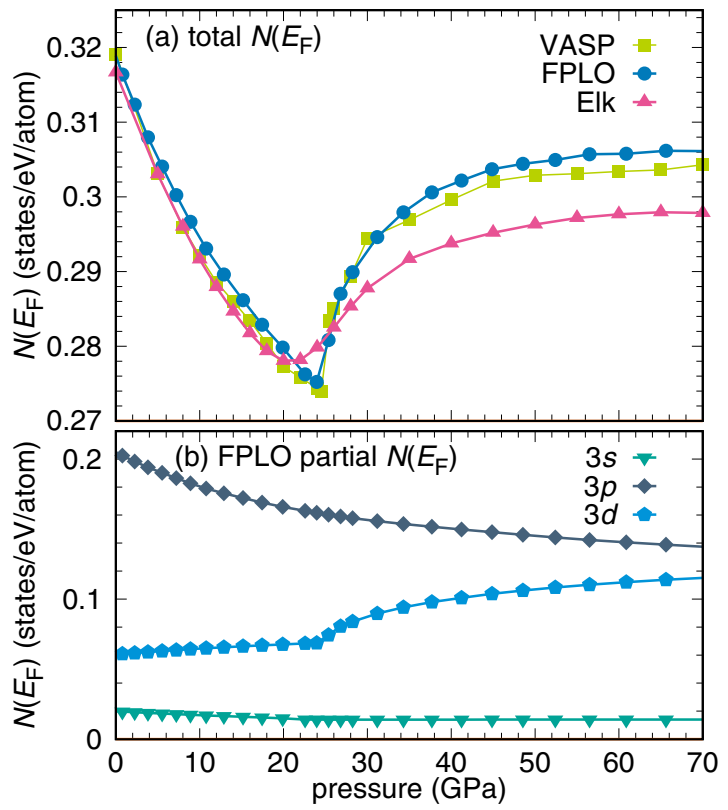

FIG. 3. Density of states at the Fermi level for the simple cubic phase as a function of external pressure. (a) Comparison between different basis sets. (b) Orbital contributions to $N\left(E_{\mathrm{F}}\right)$.
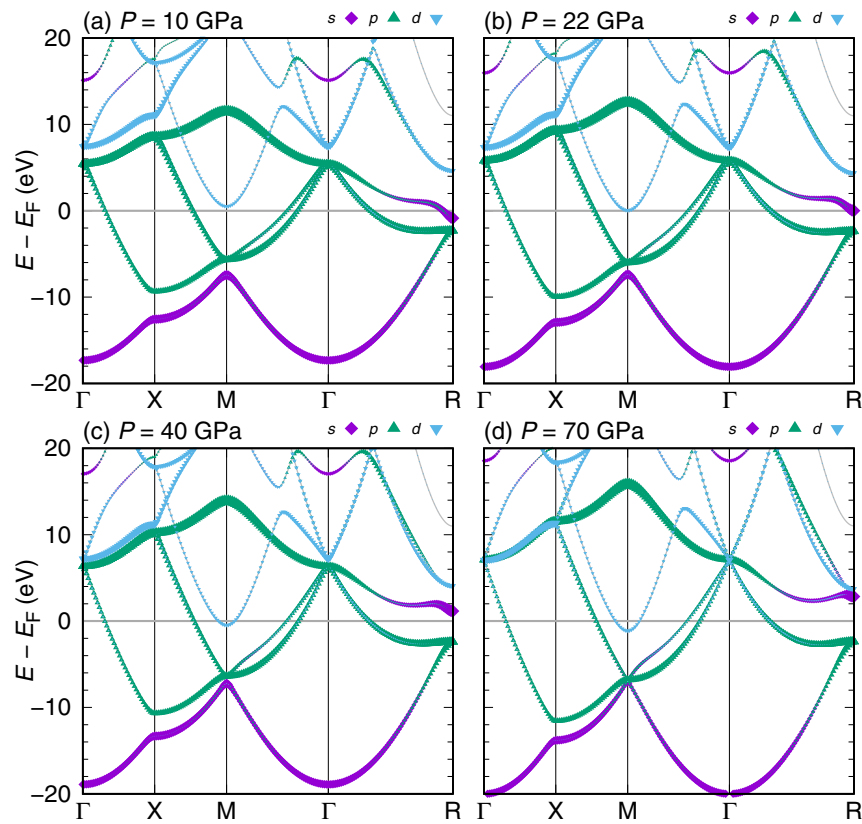

FIG. 4. Band structures with orbital weights for simple cubic phosphorus at four different external pressures.

We find that $N\left(E_{\mathrm{F}}\right)$ first decreases linearly, and then increases with the increase of external pressure, which results in a local minimum in $N\left(E_{\mathrm{F}}\right)$. The corresponding pressure is $22 \mathrm{GPa}$ $(a=2.332 \AA)$ in PBE calculations. The $N\left(E_{\mathrm{F}}\right)$ we obtain as function of pressure in principle compares well with previous calculations $[17,21]$ but has a more pronounced minimum. The orbitally resolved $N\left(E_{\mathrm{F}}\right)$ in Fig. 3(b) shows that the $3 d$ orbital is responsible for the increase of $N\left(E_{\mathrm{F}}\right)$ beyond the minimum at $P=22 \mathrm{GPa}$; taking only $s$ and $p$ orbitals into account, $N\left(E_{\mathrm{F}}\right)$ would fall monotonously.

To explain the observed changes in the DOS, we plot the orbital-projected band structures for external pressures of $P=10,22,40$, and $70 \mathrm{GPa}$ in Fig. 4. We find that the major changes at the Fermi level occur in bands with $s$ and $d$, rather than $p$ character. At the $R$ point, a band with $s$ character which is partially occupied below $22 \mathrm{GPa}$ shifts up in energy with increasing pressure, while a band around $M$ with $d$ character changes with pressure in an opposite fashion, and starts to become populated around $P=22 \mathrm{GPa}$. These changes are consistent with previous calculations [17,18,21]. The corresponding Fermi surfaces (FSs) at these pressures are shown in Fig. 5. At $10 \mathrm{GPa}$, close to the rhombohedral simple cubic phase transition point in experiment, there are three FSs: a cubic hole FS $\alpha$ around $\Gamma$, a big open-type FS $\beta$, and a small spherical electron FS $\gamma$. The $\alpha$ and $\beta$ FSs are attributed to $p$ orbitals and enclose about 0.07 holes and 1.068 electrons (or 0.932 holes), respectively. The band contributing to the $\beta$ FS is close to half-filling. The $\gamma$ FS, predominantly formed by $s$ orbitals, encloses about 0.002 electrons. As pressure increases, the $\gamma$ FS gradually shrinks and the $\beta$ FS grows a bit while the $\alpha$ FS remains almost unchanged. At $P=22 \mathrm{GPa}$, the $\gamma$ FSs disappear completely, and the Lifshitz transition occurs. With a slight further increase of pressure, three ellipsoidal electron FSs $\delta$ appear around $M$. Two Lifshitz transitions 


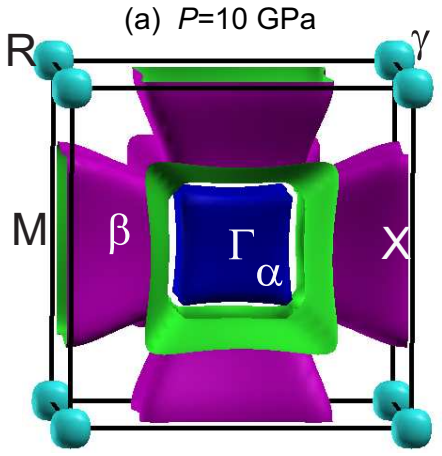

(c) $P=40 \mathrm{GPa}$

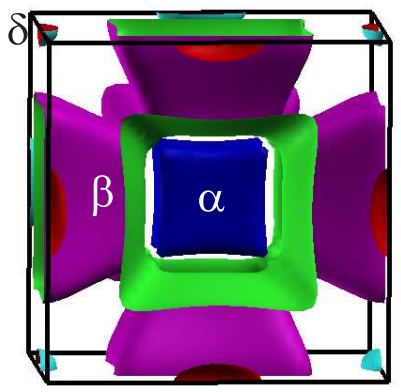

FIG. 5. The Fermi surfaces for external pressures $P=10$ (a), 22 (b), 40 (c), and $70 \mathrm{GPa}(\mathrm{d})$.

happen almost at the same pressure and, as it turns out, have a significant effect on the e-ph coupling. The $\delta$ FSs are of $d$-orbital character, as expected from the band structures in Fig. 4. As correlation effects in $d$ orbitals are stronger as compared to $p$ orbitals, the appearance of the $\delta$ FSs around $M$ may trigger stronger electronic correlation effects. This runs contrary to the expectation that pressure increases the bandwidth, and thus should decrease correlation effects as a matter of principle. To investigate the correlation effects, we performed calculations with hybrid functional and GGA $+U$. The band structures are given in Appendix A. In calculations with the hybrid functional, the bandwidth increases and the most noticeable changes near the Fermi level are that the $s$ band at $R$ and the $d$ band at $M$ are are shifted to slightly higher energy, and as a consequence the two Lifshitz transitions happen separately. In GGA $+U$ calculations, however, the obtained band structures are almost unchanged compared to the GGA functional. Therefore, correlations have little effect on the band structure, but may still have a significant effect on the superconductivity of phosphorus.

\section{B. Electron-phonon coupling for cubic phosphorus}

Now, we turn to the effect of pressure on the phonons for simple cubic phosphorus. The phonon spectra and densities of states are shown in Fig. 6 for several pressures. Below $P=16 \mathrm{GPa}$, there are imaginary phonon modes around the $R$ point. The unstable modes correspond to the lattice distortion in the direction of the rhombohedral $A 7$ phase [21]. Above $P=16 \mathrm{GPa}$, unstable phonon modes vanish and most phonon states lie in two regions: a low-energy region (LER) and a high-energy region (HER). DOS is small in the LER but shows large peaks in the HER; the latter contains more states than

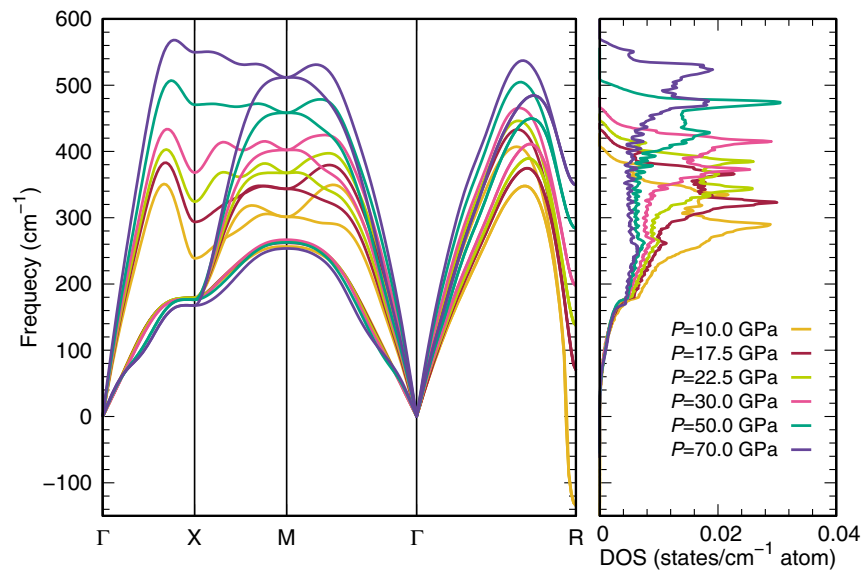

FIG. 6. Phonon spectra and densities of states for simple cubic phosphorus at several pressures.

the former. At 17.5 GPa, for example, the LER is from 180 to $280 \mathrm{~cm}^{-1}$ and the HER is from 280 to $400 \mathrm{~cm}^{-1}$. In the LER, phonon bands are relatively smooth, and phonon states are mainly due to phonon modes around $\Gamma$ and $R$ as well as low-energy modes around $X$ and $M$. In the HER, the situation is quite different. Along the $\Gamma-X$ direction, the frequency of the longitudinal mode increases and reaches a maximum near the $X$ point, to then drop suddenly. One mode along $\Gamma-M$ and three modes along $\Gamma-R$ direction exhibit similar behavior, and the drops near the $R$ point are the largest. All this finally results in a large DOS in the HER. As pressure increases, the drops near $X, M$, and $R$ decrease and most phonon modes harden, which leads to the widening of the LER and a shift towards higher frequency for the HER. These changes have a significant effect on the e-ph coupling discussed in the following.

To investigate the e-ph coupling for simple cubic phosphorus, we first plot the phonon linewidth at two instructive values of pressure (before and after the Lifshitz transitions) in Fig. 7. Strong e-ph coupling occurs in the high-energy modes around $X$ and $M$ and all modes around $R$, which is due to the large Fermi-surface nesting at these $\boldsymbol{q}$ vectors [21]. With the increase of pressure, the phonon linewidth near $X, M$, and $R$ increases a little bit. The obtained $\lambda_{\nu q}$ along high-symmetry $k$ points for four pressures are shown in Fig. 8. The e-ph coupling constant $\lambda_{\nu \boldsymbol{q}}$ is peaked at $R$. It is rather different from the distribution of phonon linewidths $\gamma_{\nu \boldsymbol{q}}$, where $\gamma_{3 M}$ and $\gamma_{3 X}$ are comparable with $\gamma_{R}$. As the phonon frequencies at $R$ are lower than those at $X$ and $M$, and since $\lambda_{v \boldsymbol{q}}$ is inversely proportional to $\omega_{v \boldsymbol{q}}^{2}$, the $\lambda_{X}$ and $\lambda_{M}$ are much smaller than $\lambda_{R}$. Most $\lambda_{v \boldsymbol{q}}$ decrease with increasing pressure. However, contrary to the pressure-dependent behavior of $\lambda_{\nu \boldsymbol{q}}$ around $X, M$, and $R$, after the Lifshitz transitions the $\lambda_{1 q}$ and $\lambda_{2 q}$ around $\Gamma$ (along $\Gamma-X, \Gamma-M$, and $\Gamma-R$ directions) increase with increasing pressure, which can be attributed to the softening of phonon modes at these $\boldsymbol{q}$ vectors. These features are consistent with the previous plane-wave pseudopotential calculation [21].

In order to analyze the contributions of different Fermi surfaces to the e-ph coupling, we plot the band-resolved Eliashberg spectral functions in Fig. 9. Before the Lifshitz 
(a) $P=17.5 \mathrm{GPa}$

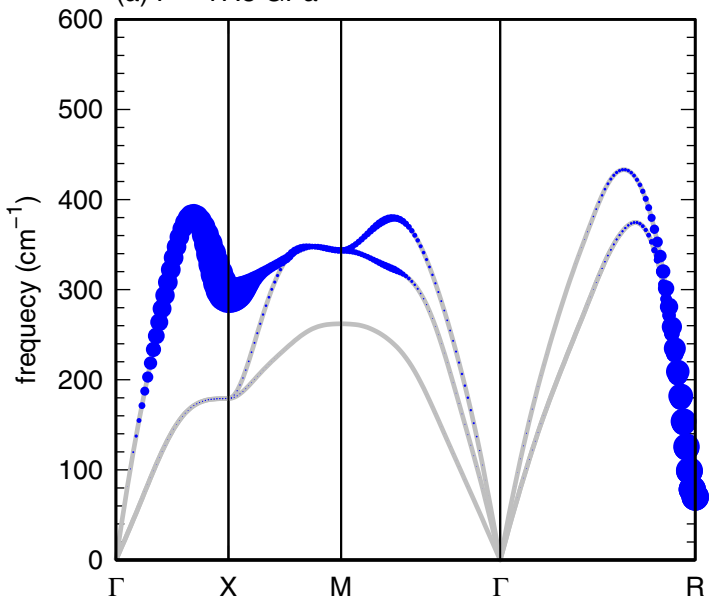

(b) $P=30 \mathrm{GPa}$

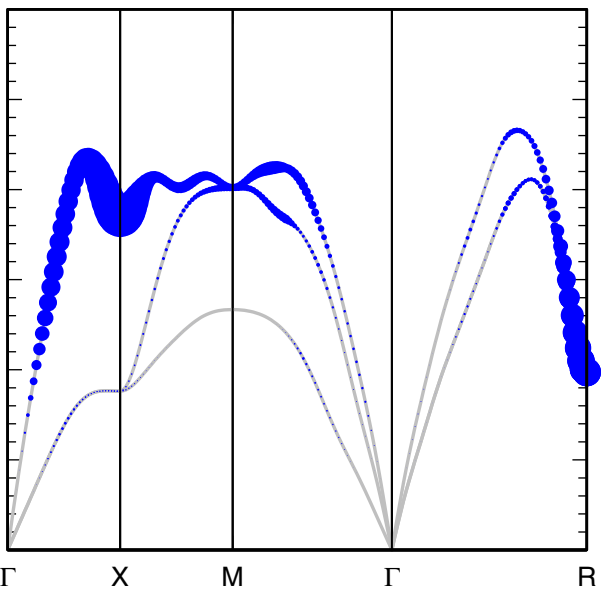

FIG. 7. The phonon linewidth $\gamma_{v q}$ along high-symmetry $q$ points at $P=17.5 \mathrm{GPa}$ (before the Lifshitz transition) and $P=30 \mathrm{GPa}$ (after the Lifshitz transition). The sizes of the circles represent the magnitude of $\gamma_{v q}$.

transition, at $P=17.5 \mathrm{GPa}$ the $\beta \beta$ intraband coupling and $\alpha \beta$ interband coupling contribute dominantly to e-ph coupling, which is due to the large- $\beta$ Fermi surfaces and relative good nesting between $\alpha$ and $\beta$ Fermi surfaces. As the spherical $\gamma$ FS is well separated from $\alpha$ and $\beta$ FS in $k$ space, the $\alpha \gamma$ and $\beta \gamma$ interband couplings are relatively weak. Upon increasing pressure, the peak of $\beta \beta$ intraband coupling becomes broad and shifts to higher frequency, which results in a significant e-ph coupling decrease in the $\beta \beta$ channel. At the Lifshitz transition point, the $\alpha \gamma$ and $\beta \gamma$ interband couplings vanish due to the disappearance of the $\gamma$ FS. Thus, e-ph coupling decreases with increasing pressure before the Lifshitz transition, as shown in Fig. 10. As pressure further increases, three $\delta$ FSs with $d$ orbital character appear around the $M$ point, and they introduce strong interband couplings in $\alpha \delta$ and $\beta \delta$ channels [see Fig. 9(c)], similar to the case of sulfur under pressure [43]. The e-ph coupling increases with increasing pressure because the e-ph coupling enhancements from new channels overcome the weakening of couplings in $\alpha \beta$ and $\beta \beta$ channels. Therefore, $\lambda$ exhibits a valley around the Lifshitz transition point. After the

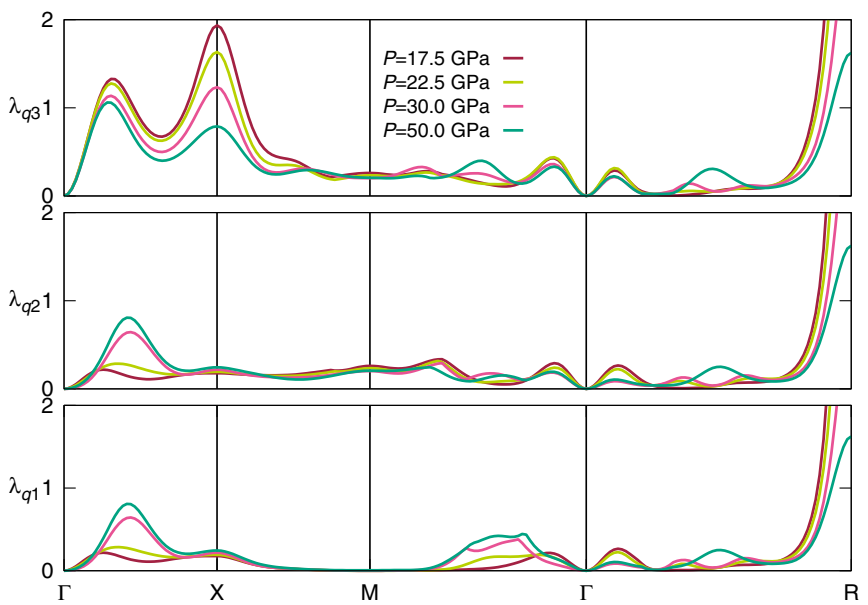

FIG. 8. Electron-phonon coupling constant $\lambda_{\nu q}$ along highsymmetry $q$ directions at $P=17.5,22.5,30.0$, and $50.0 \mathrm{GPa}$.
Lifshitz point, the isotropic $\lambda$ increases and reaches a maximum at $P=30 \mathrm{GPa}$. As pressure further increases, the two broad peaks in $\beta \delta$ channels show opposite behaviors: one shifts to higher frequency and the other shifts to lower frequency. The remaining interband and intraband e-ph couplings shift to higher frequency. This will lead to decreased e-ph couplings in most channels except the $\delta \delta$ intraband channel. Therefore, $\lambda$ will decrease again.

The isotropic Eliashberg spectral functions $\alpha^{2} F(\omega)$ at five selected pressures are shown in Fig. 11. We find that the $\alpha^{2} F(\omega)$ have almost the same shapes as the phonon DOS shown in Fig. 6. The e-ph coupling occurs mainly in the highenergy region and it is mainly due to $\beta \beta$ intraband coupling and $\alpha \beta, \alpha \delta$, and $\beta \delta$ interband coupling. This high-energy spectral
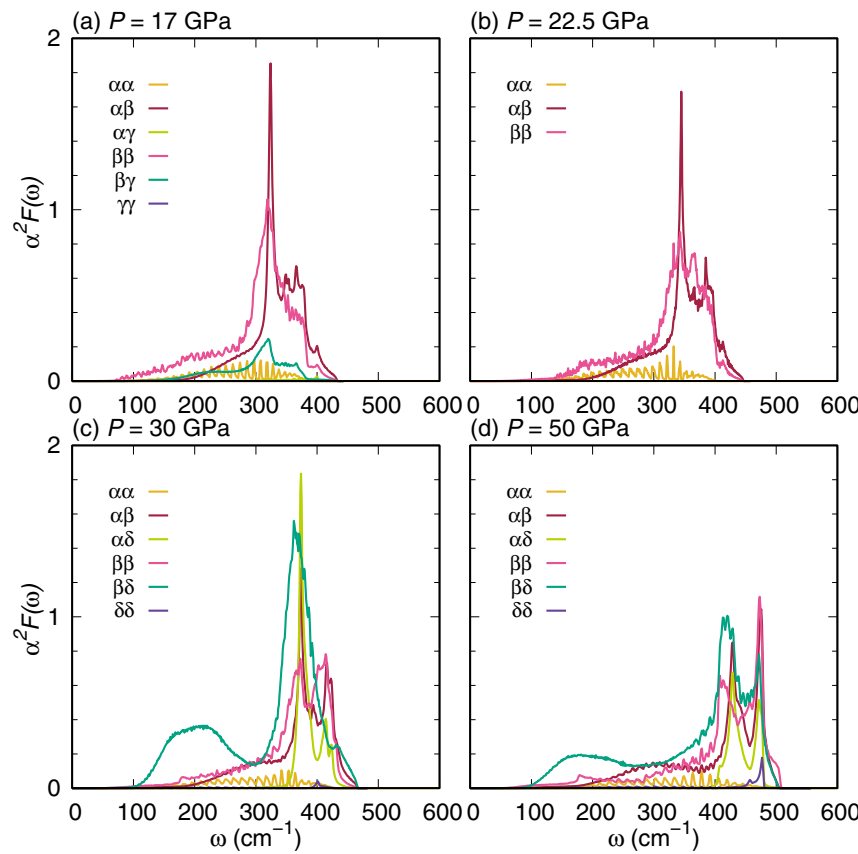

FIG. 9. Band-resolved Eliashberg spectral function $\alpha^{2} F_{m n}(\omega)$ for cubic phosphorus at $P=17.5,22.5,30.0$, and $50.0 \mathrm{GPa}$. 


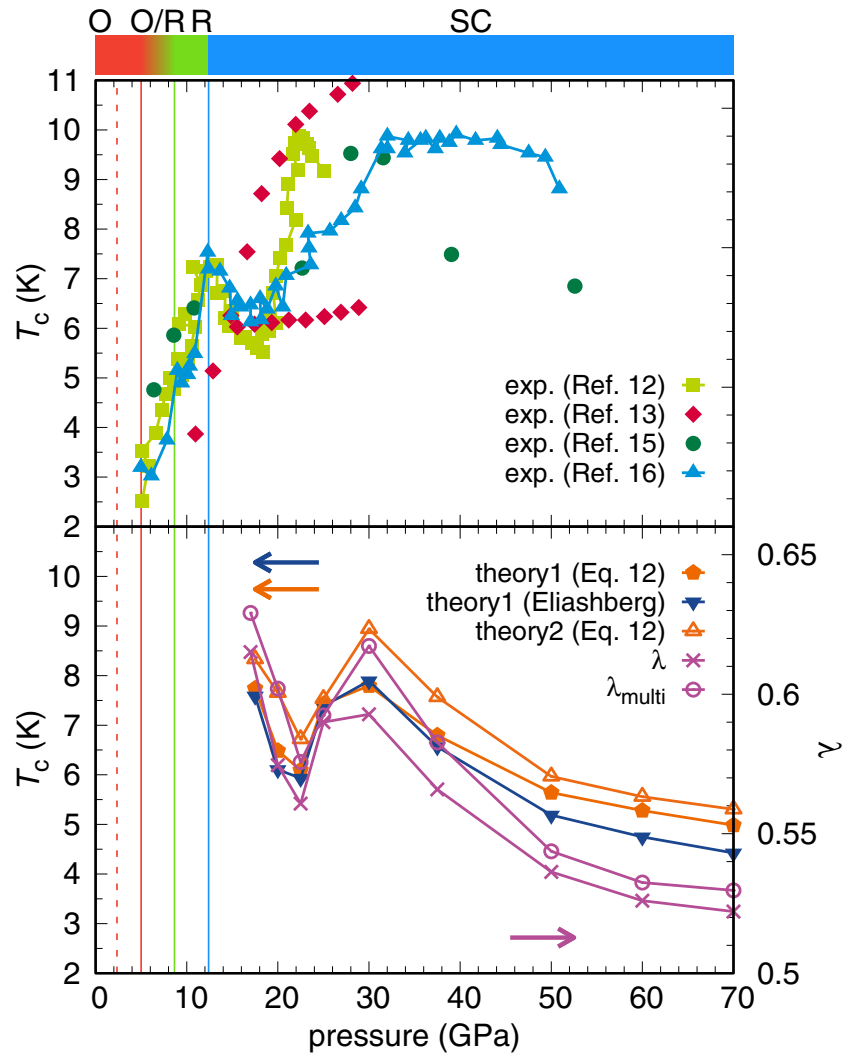

FIG. 10. Superconducting transition temperature $T_{c}$ and e-ph coupling constant $\lambda$ as a function of pressure in theoretical calculations compared to the experimental data. Squares, circles, diamonds, and triangles denote the data from J. Wittig et al. [12], Karuzawa et al. [15], Kawamura et al. [13], and Guo et al. [16], respectively. "Theory 1" denotes $T_{c}$ from the isotropic $\lambda$ and "Theory 2 " denotes $T_{c}$ from the multiband $\lambda_{\text {multi }}$.

weight shifts to higher frequency with increasing pressure, rather similar to the behavior of the phonon DOS. Another noticeable feature in $\alpha^{2} F(\omega)$ is that the e-ph coupling (100 to $200 \mathrm{~cm}^{-1}$ ) in the low-energy region increases as pressure

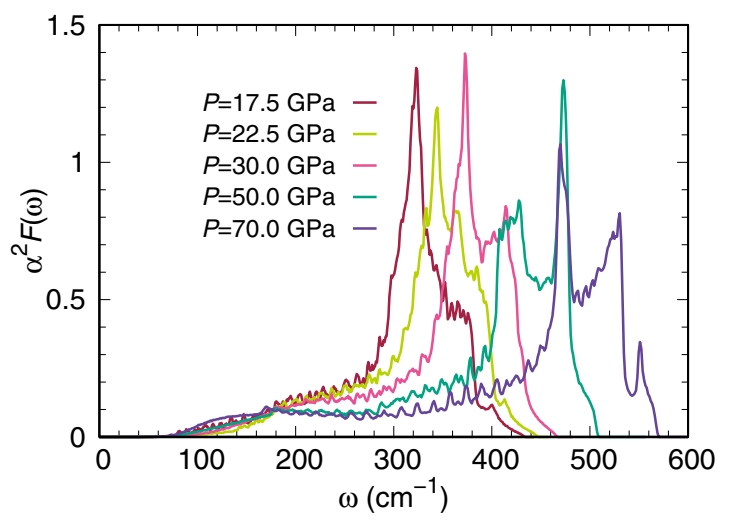

FIG. 11. Eliashberg spectral function $\alpha^{2} F(\omega)$ for cubic phosphorus at $P=17.5 \mathrm{GPa}(a=2.350 \AA), 22.5 \mathrm{GPa}(a=2.330 \AA)$, $30.0 \mathrm{GPa}(a=2.300 \AA), 50.0 \mathrm{GPa}(a=2.246 \AA)$, and $70.0 \mathrm{GPa}$ $(a=2.201 \AA)$. increases, which can be mainly attributed to e-ph coupling in the $\beta \delta$ channel.

We calculate $T_{c}$ using the McMillan equation [Eq. (12)] and the Coulomb pseudopotential parameter $\mu^{*}$ is fixed to $\mu^{*}=0.12$, a value for $\mu^{*}$ that matches the minimum $T_{c}$ in our calculations to the experimentally determined $T_{c}$ valley observed in Ref. [16]. This $\mu^{*}$ is significantly smaller than that in Ref. [21]. We also calculate $T_{c}$ by solving the isotropic Eliashberg equations self-consistently [28]. Figure 10 shows the calculated isotropic e-ph coupling constant $\lambda$, multiband e-ph coupling constant $\lambda_{\text {multi }}$, and $T_{c}$ as a function of pressure in theory, compared to the experimental data from different groups. The multiband e-ph coupling constant and $T_{c}$ are larger than the corresponding isotropic ones. With increasing pressure, the isotropic and multiband $\lambda$ and $T_{c}$ first decrease and then increase, thus form a valley, and finally decrease again. We also performed calculations using EPW, where the trend of pressure dependent $\lambda$ and $T_{c}$ turns out to be rather similar (see Appendix B). The obtained $\lambda$ are close to those in Refs. [17-19] but smaller than those in Ref. [21]. The origin of this difference is unclear, and may be related to an inherent difference of the approaches. Overall, the trend as a function of pressure is not consistent with the recent e-ph coupling calculations using a Wannier interpolation approach [21], where a large smearing parameter $\sigma(0.27 \mathrm{eV})$ is adopted which significantly overestimates $T_{c}$ before the Lifshitz transitions.

From our theory, the calculated $T_{c}$ exhibits a valley formation similar to Ref. [16] depicted as down triangles in Fig. 10, as it is also consistent with the data in Ref. [12] (green squares) in the range from $P=5$ to $25 \mathrm{GPa}$. While our calculations can thus explain the observed $T_{c}$ valley, the most prominent feature in recent experiments, they do not explain the plateau seen in Ref. [16] beyond $P=32 \mathrm{GPa}$. The $T_{c}$ valley can be attributed to the Lifshitz transitions, which is also consistent with the Hall measurements [16]. The pressure for the $T_{c}$ minimum in experiment and our theoretical calculations are at 17 and $22 \mathrm{GPa}$, respectively. The difference is relatively small, and can be decreased if we include correlation effects in the calculations. Two state-of-the-art methods, pseudopotential calculations combined with Wannier function interpolation and full potential all-electron calculations, arrive at the same result which can successfully explain the $T_{c}$ valley formation.

\section{DISCUSSION}

Above $5 \mathrm{GPa}$, when orthorhombic black phosphorus partially converts to the rhombohedral phase, superconductivity appears. In the mixed phase region, $T_{c}$ increases. At $12 \mathrm{GPa}$, the rhombohedral phase transforms into the cubic phase, and $T_{c}$ shows a jump [16]. Our calculations indicate that the phase transition happens at $19 \mathrm{GPa}$ (see Appendix C), which is higher than that in experiment. Moreover, the couplings between $p$ orbitals along [111] direction in the cubic phase are uniform compared with those in the rhombohedral phase due to its high-symmetry structure, which suppresses band splitting near the Fermi level, and hence results in a higher $N\left(E_{f}\right)$. Therefore, $N\left(E_{f}\right)$ shows a jump across the structural phase transitions, which directly feeds into the value of $T_{c}$. Furthermore, we perform phonon calculations in both phases at $22 \mathrm{GPa}$, where $d$-orbital bands are still not involved. The 


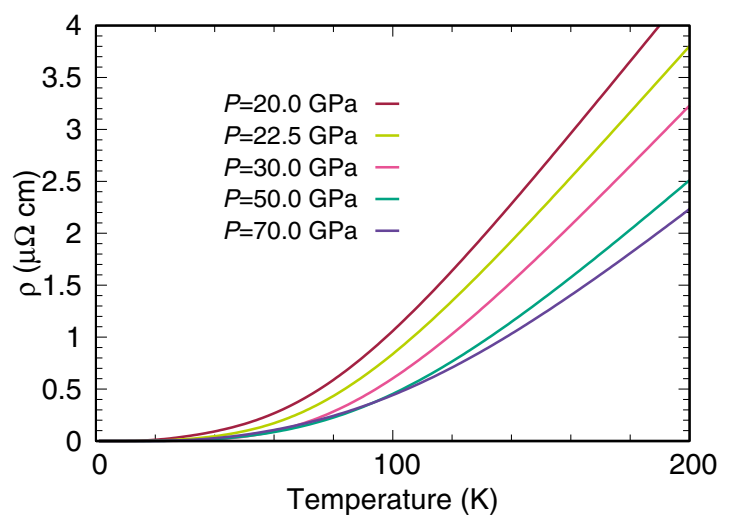

FIG. 12. Temperature-dependent resistivity for cubic phosphorus from e-ph coupling.

phonon spectra are given in Appendix C. Due to the change in crystal symmetry at the phase transition, in the cubic phase there are more degenerate phonon modes and the phonons soften a little compared with the rhombohedral phase. This leads to an increased e-ph coupling constant and $T_{c}$ shows a jump of about $1.3 \mathrm{~K}$ with $\mu^{*}=0.12$. It is consistent with experimental data in Ref. [16], which shows that the structural phase transition taking place at $12.4 \mathrm{GPa}$ is accompanied by a jump in $T_{c}$. At this low pressure, the Lifshitz transition cannot occur according to our calculations. Therefore, the $T_{c}$ jump across the phase transition should be mainly attributed to the change in crystal symmetry rather than the Lifshitz transition.

In the normal state, simple cubic phosphorus exhibits a quadratic temperature dependence of the resistance in the normal state, and resistance decreases as pressure increases [16]. The resistance due to e-ph coupling can be expressed as [44]

$$
\rho(T)=\frac{4 \pi m_{e}}{n e^{2} k_{B} T} \int_{0}^{\infty} d \omega \hbar \omega \alpha_{\mathrm{tr}}^{2} F(\omega) n(\omega, T)[1+n(\omega, T)],
$$

where $n(\omega, T)$ is the Bose-Einstein distribution. The Eliashberg transport coupling function is similarly defined as the Eliashberg spectral function [44]. According to our calculations, the shape of $\alpha_{\text {tr }}^{2} F(\omega)$ is very similar to that of $\alpha^{2} F(\omega)$, and with increasing pressure $\alpha_{\mathrm{tr}}^{2} F(\omega)$ exhibits similar behavior compared to $\alpha^{2} F(\omega)$. It will lead to a decreased resistance with increasing pressure, as shown in Fig. 12, which is consistent with the experimental data both in its temperature and its pressure dependence. This suggests that e-ph coupling has a significant contribution to the electrical resistance.

Our calculations can successfully explain the observed $T_{c}$ valley in the simple cubic phase but not the $T_{c}$ plateau at high pressure. The $T_{c}$ valley in experiment turns out to have an electronic origin, as it is due to Lifshitz transitions. At high pressure, the phonon modes harden rapidly, and the e-ph coupling constant as well as $T_{c}$ decrease with increasing pressure in our calculations, which is inconsistent with the observed $T_{c}$ plateau at a rather high value. If we study how $T_{c}$ evolves in experiment for higher pressure, one finds that $T_{c}$ remains almost unchanged for an extended regime of varying pressure, while the hole carrier density decreases for
$P>30 \mathrm{GPa}$. Such evidence is rather intriguing, and hard to explain. Let us first note that systematic errors in the actual pressure imposed on the crystal might in fact be bigger for higher than for lower pressures. As a consequence, it potentially cannot be excluded that the increased pressure is not homogeneously imposed on the whole crystal, and that the plateau might be in part attributed to that. Instead, starting from the hypothesis that we take this precise shape of $T_{c}$ as reference, one possible explanation could be that electronic correlations may contribute to superconductivity in simple cubic phosphorus at high pressure. From the analysis above, we know that electron pockets around the $M$ points are mostly composed of $d$ orbitals, and appear when $P>22 \mathrm{GPa}$. The electronic correlation effects in $d$ orbitals are expected to be stronger than those in $p$ and $s$ orbitals. As such, these correlation effects should become more and more important at high pressures, even though the overall bandwidth increases. Assuming that electron-electron interactions might participate in the pairing mechanism on these Fermi surfaces, with increasing pressure, the $\delta$ Fermi surfaces grow bigger, and the carrier density of electrons with $d$-orbital character increases (see Fig. 2), which could enhance the Cooper pairing. This will further enlarge the pressure region where $T_{c}$ increases. If the enhancement from correlation effects can make up for the $T_{c}$ decrease due to phonon hardening, it may lead to such a $T_{c}$ plateau at high pressure. In Ref. [22], where electronic interactions have been considered within the static random phase approximation (RPA), one could not reproduce the experimentally observed behavior of $T_{c}$ at high pressure. This suggests that effects which are beyond the static RPA level need to be taken into account. One possibility is that the frequency dependence of the interaction is important, and that plasmons, which can cooperate with phonons and significantly raise $T_{c}$, provide an explanation [45].

\section{CONCLUSIONS}

We have investigated the electronic structure and electronphonon coupling for simple cubic phosphorus by performing first-principles calculations within the FP-LAPW method. Our calculations show that with increasing pressure, $T_{c}$ first decreases, then increases and so forms a valley, and then decreases again. Before the Lifshitz transitions set in, the phonon hardening and shrinking of the $\gamma$ Fermi surface result in a decrease of $T_{c}$ with increasing pressure. After the Lifshitz transitions, the appearance of $\delta$ Fermi surfaces with $3 d$ orbital character generates strong electron-phonon inter-band couplings in $\alpha \delta$ and $\beta \delta$ channels, and hence increases $T_{c}$. With further increase of pressure, phonon hardening makes a dominant contribution to electron-phonon coupling, and $T_{c}$ decreases again. Our study reveals that the intriguing $T_{c}$ valley, as found in experiment, should be attributed to the Lifshitz transitions. We also find, however, that the experimentally observed $T_{c}$ plateau at high pressure is beyond the electronphonon mechanism considered here. It suggests that aside from electron-phonon coupling, plasmonic contributions along with electronic correlations may be relevant for systems with $d$-orbital character at higher pressures, such as for simple cubic black phosphorus. 


\section{ACKNOWLEDGMENTS}

We particularly thank G. Profeta for highly useful discussions of the electron-phonon coupling for multiband systems. We also thank P. Barone, L. Boeri, M. Gao, C. Verdi, K. Dewhurst, G. Sangiovanni, and G. Li for illuminating discussions. We also thank L. Sun and H. Wang for helpful discussions on the experimental aspects. We acknowledge the Gauss Centre for Supercomputing e.V. (www.gauss-centre.eu) for funding this project by providing computing time on the GCS Supercomputer SuperMUC at Leibniz Supercomputing Centre (LRZ, www.lrz.de). This work has been supported by Grants No. ERC-StG-TOPOLECTRICS-336012, No. SFB 1170 TOCOTRONICS, and No. SPP 1666. The work at Princeton was supported by the Gordon and Betty Moore Foundation EPiQS initiative, Grant No. GBMF-4412. The work in Zurich was supported by the Swiss National Science Foundation under Grant No. PZ00P2_174015.

\section{APPENDIX A: BAND STRUCTURES FOR SIMPLE CUBIC PHOSPHORUS WITH GGA $+U$ AND HSE}

According to our calculations, a band with $d$-orbital character will cross the Fermi level at sufficiently high pressure. This suggests that correlation effects may be important in simple cubic phosphorus, and we check if they have an important effect on the electronic structure. First, we perform calculations with a hybrid functional (HSE06) [46]; the obtained band structures are shown in Fig. 13 in comparison with GGA bands. Compared to the bands calculated with GGA functional, the bandwidth in the HSE bands increases, and the most noticeable changes near the Fermi level are that the $s$ band at $R$ and the $d$ band at $M$ are are shifted to slightly higher energies. The two Lifshitz transitions at the $R$ and $M$ points occur at almost the
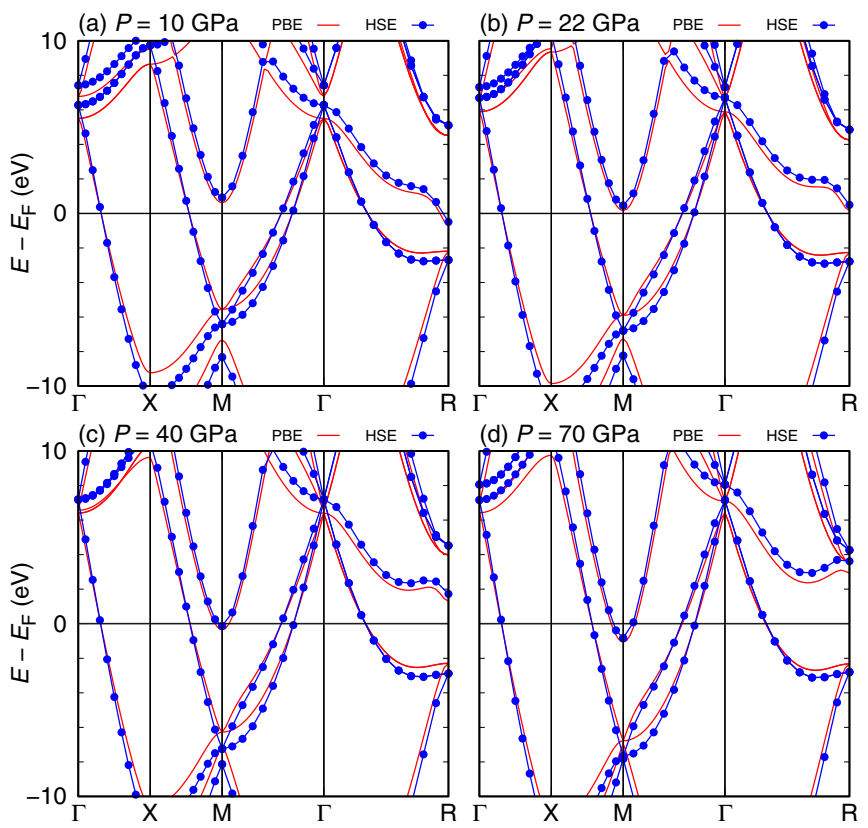

FIG. 13. Band structures with GGA functional and hybrid functional (HSE06) for simple cubic phosphorus at four different external pressures.
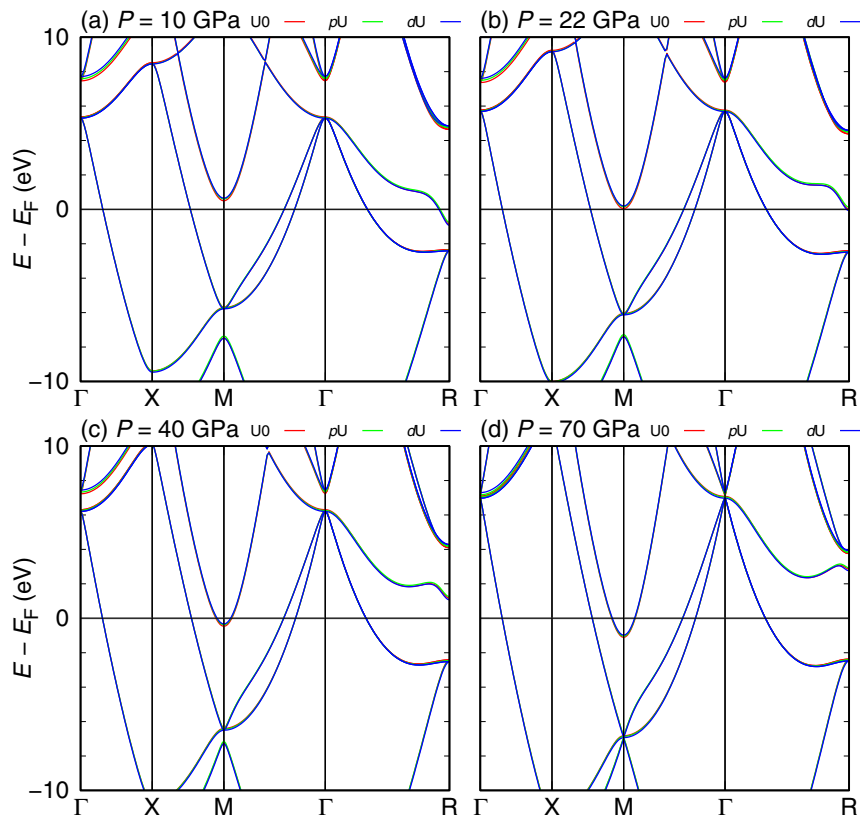

FIG. 14. The band structures with GGA $+U$ for simple cubic phosphorus at four different external pressures. $U 0$ represents GGA calculations, $p U$ and $d U$ indicate that in the GGA $+U$ calculations the additional $U$ is on $p$ orbital and $d$ orbital, respectively.

same pressure in GGA calculations but happen at 20 and 38 $\mathrm{GPa}$ in HSE calculations.

We also tried to consider the local interaction by performing a GGA $+U$ calculation. The adopted $U$ and $J$ are 2.0 and $0.5 \mathrm{eV}$ for $p$ and $d$ orbitals, respectively. The band structures are given in Fig. 14. No matter whether the additional $U$ interaction is on $p$ or $d$ orbitals, the obtained band structures are almost the same as those with GGA functional. Therefore, GGA $+U$ has little effect on the band structures of simple cubic phosphorus. Furthermore, we calculate the phonon spectra for phosphorus at 20 and $25 \mathrm{GPa}$, and the phonon dispersions are shown in Fig. 15. The phonon modes in GGA $+U$ calculations soften a little bit compared with those in GGA calculations. This phonon softening will increase the

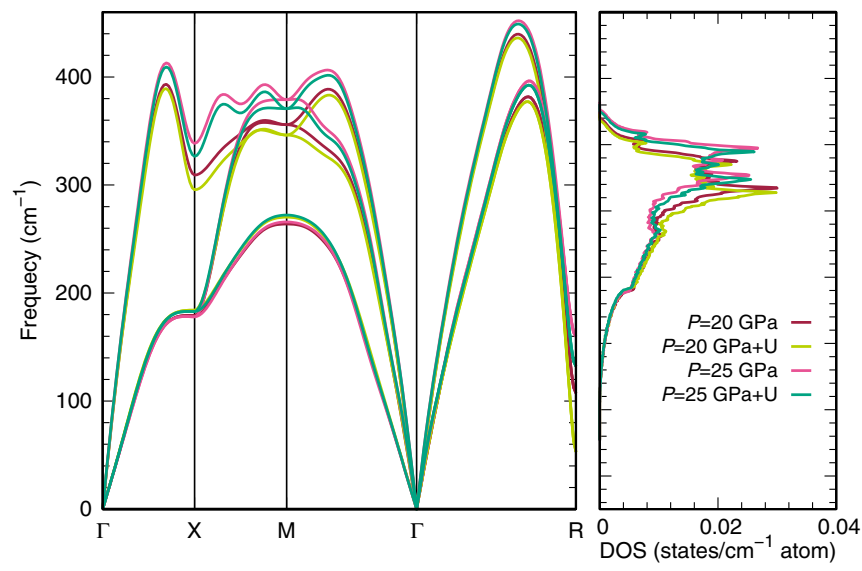

FIG. 15. Phonon spectra and densities of states with GGA and $\mathrm{GGA}+U$ for simple cubic phosphorus at $P=20$ and $25 \mathrm{GPa}$. 


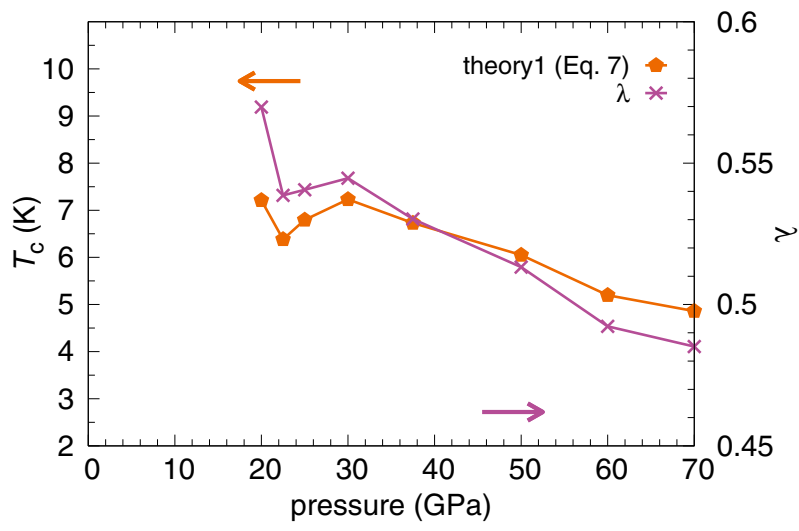

FIG. 16. Superconducting transition temperature $T_{c}$ and e-ph coupling constant $\lambda$ as a function of pressure in theoretical calculations using EPW. The adopted $\mu^{*}$ is 0.1 .

e-ph coupling but cannot change the general negative trend of the pressure-dependent e-ph coupling constant.

\section{APPENDIX B: ELECTRON-PHONON COUPLING CONSTANT AND $T_{c}$ FROM EPW}

We also perform electron-phonon coupling calculations using EPW. To obtain the nine Wannier functions, we use the Bloch functions on an $8 \times 8 \times 8 k$ mesh. Phonon dispersions are calculated on an $8 \times 8 \times 8 q$ mesh using density functional perturbation theory. For the e-ph calculations, a very fine $128 \times 128 \times 128 k$ mesh and a $24 \times 24 \times 24 q$ mesh are used, and the $\delta$ function in the phonon linewidth is approximated by Gaussian functions with $\sigma=0.08 \mathrm{eV}$. After obtaining good Wannier functions, which can be used to reproduce the first-principles band structures and phonon dispersions with high accuracy, we perform electron-phonon calculations. The obtained electron-phonon coupling constant and $T_{c}$ are shown in Fig. 16. Similar to the results from ELK, both $\lambda$ and $T_{c}$ first decrease then increase, forming a valley, and decrease again with increasing pressure. Therefore, both ELK and EPW calculations confirm that there is a $T_{c}$ valley in the superconducting $T_{c}$ versus pressure phase diagram, which can be attributed to the Lifshitz transitions. The results are consistent with those from ELK calculations.

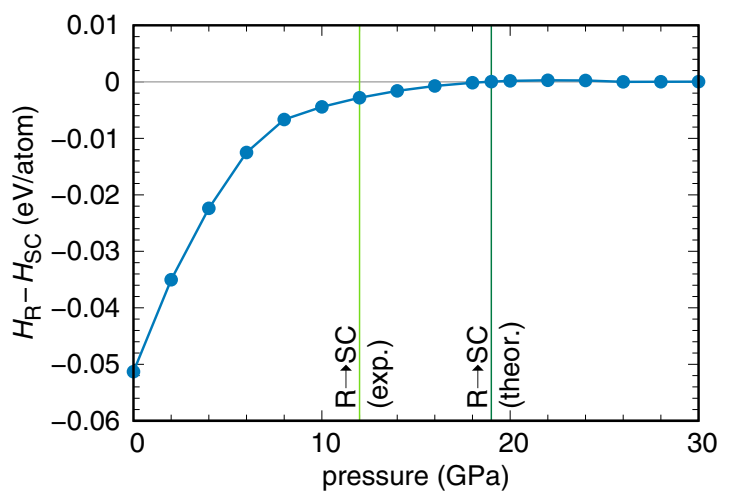

FIG. 17. Enthalpy for rhombohedral phosphorus relative to the simple cubic phase as a function of pressure.

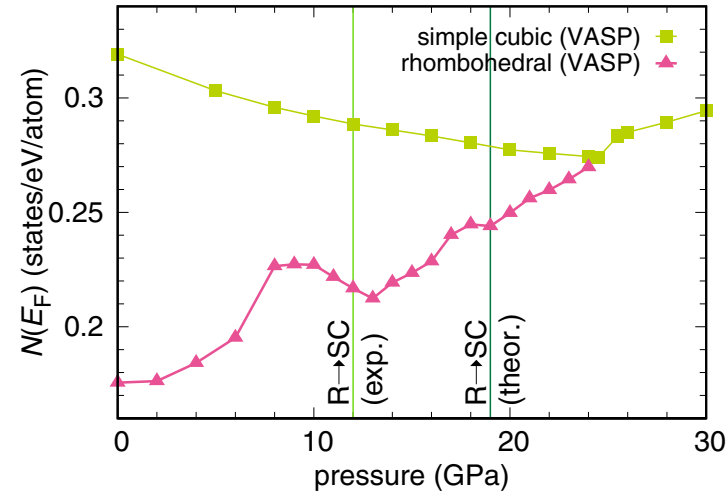

FIG. 18. Density of states at the Fermi level for the simple cubic and rhombohedral phases as a function of pressure.

\section{APPENDIX C: DENSITY OF STATES EVOLUTION FROM RHOMBOHEDRAL TO CUBIC PHOSPHORUS}

While we have focused on simple cubic phosphorus in this work as it is the phase with the highest superconducting transition temperature, it is also interesting how the transition to the high- $T_{c}$ phase occurs. We first investigate the stability of rhombohedral phosphorus relative to simple cubic phosphorus by calculating enthalpies as a function of external pressure; the difference between enthalpies in simple cubic and rhombohedral phases is shown in Fig. 17. Below 9 Gpa, the dominant structure is orthorhombic, but here we focus on the cubic and rhombohedral phases above $10 \mathrm{GPa}$. From our calculations, the enthalpies of the two phases are very close above $10 \mathrm{GPa}$ and the rhombohedral to simple cubic phase transition occurs at $19 \mathrm{GPa}$, which is consistent with previous calculations [21]. This transition pressure is higher than the $12 \mathrm{GPa}$ observed in experiment [16]. Figure 18 shows the DOS at the Fermi level for simple cubic and rhombohedral phases as a function of pressure. At the phase transition, the density of states at the Fermi level $N\left(E_{F}\right)$ jumps up sharply (both at the experimental and at the theoretical transition pressures). As $T_{c}$ is often proportional to $e^{-\frac{1}{V N\left(E_{F}\right)}}$, the jump of $N\left(E_{F}\right)$ can lead to a jump in $T_{c}$, which explains the observed $T_{c}$ jump across the phase transition in experiments [16]. Moreover, the $T_{c}$ increase from 20 to $25 \mathrm{GPa}$ in Ref. [22] should partially be due to the $N\left(E_{F}\right)$ increase. However, $\mathrm{x}$-ray diffraction measurements show that the cubic phase is pure above $13.44 \mathrm{GPa}$ [16]. The adopted
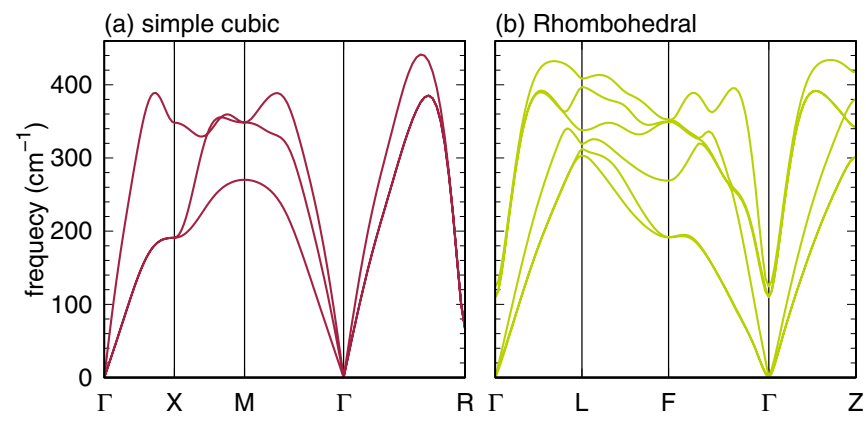

FIG. 19. Phonon spectra for the simple cubic and rhombohedral phases at $22 \mathrm{GPa}$. 


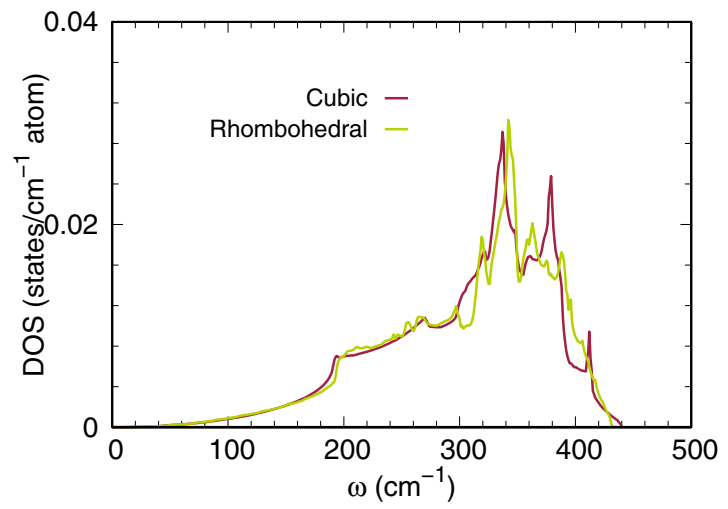

FIG. 20. Phonon density of states for the simple cubic and rhombohedral phases at $22 \mathrm{GPa}$.

structure is rhombohedral in the pressure range from 10 to $20 \mathrm{GPa}$ in calculations [22], which is not consistent with the experimental data from Guo et al. [16]. In the simple cubic phase, our conclusion is consistent with that of Ref. [22] that $T_{c}$ will decrease with increasing pressure (at high pressure).

To estimate if $T_{c}$ will jump across the phase transition, we perform calculations for phosphorus in simple cubic and rhombohedral $(R)$ phases. As there are unstable phonon modes in the cubic phase at low pressure, we perform the calculations at $22 \mathrm{GPa}$, near the theoretical phase transition point, where $N\left(E_{F}\right)$ still shows a jump. It can represent the general behavior of $T_{c}$ during the phase transition. At this pressure, the $d$ band is close to the Fermi level but does not cross it in either phase. The phonon spectra and DOS are shown in Figs. 19 and 20 and their DOS are very similar. In the $R$ phase the phonon modes harden a little and optical modes show a splitting compared with the cubic phase due to additional coupling in the lower-symmetry crystal. If we assume the same effective interaction in the two phases, the e-ph coupling constant is proportional to $N\left(E_{F}\right)$. Using the McMillan equation, we estimate that the $T_{c}$ jump is about $1.3 \mathrm{~K}$ with with $\mu^{*}=0.12$. At the lower pressure, the phonon hardening in the $R$ phase will be larger. The $T_{c}$ jump will be bigger at the experimental phase transition point because the atomic distortion in the $R$ phase is larger than that in the present calculations. Therefore, when the $R$ phase transforms into the simple cubic phase, $T_{c}$ will show a jump and it can be mainly attributed to the change of crystal symmetry (affecting the electronic structure and phonon modes) rather than to the Lifshitz transition as suggested in Ref. [22]. This is also supported by experimental data which shows that the phase transition (rhombohedral to simple cubic) happens at $12 \mathrm{GPa}[16]$ accompanied by a jump in $T_{c}$ where the Lifshitz transition cannot occur.
[1] J. C. Jamieson, Crystal structures adopted by black phosphorus at high pressures, Science 139, 1291 (1963).

[2] T. Kikegawa and H. Iwasaki, An x-ray diffraction study of lattice compression and phase transition of crystalline phosphorus, Acta Crystallogr., Sect. B: Struct. Sci. 39, 158 (1983).

[3] E. S. Reich, Phosphorene excites materials scientists, Nature (London) 506, 19 (2014).

[4] H. Liu, A. T. Neal, Z. Zhu, Z. Luo, X. F. Xu, D. Tománek, and P. D. Ye, Phosphorene: An unexplored 2D semiconductor with a high hole mobility, ACS Nano 8, 4033 (2014).

[5] L. K. Li, Y. J. Yu, G. J. Ye, Q. Q. Ge, X. D. Ou, H. Wu, D. L. Feng, X. H. Chen, and Y. B. Zhang, Black phosphorus field-effect transistors, Nat. Nanotechnol. 9, 372 (2014).

[6] Z. J. Xiang, G. J. Ye, C. Shang, B. Lei, N. Z. Wang, K. S. Yang, D. Y. Liu, F. B. Meng, X. G. Luo, L. J. Zou et al., PressureInduced Electronic Transition in Black Phosphorus, Phys. Rev. Lett. 115, 186403 (2015).

[7] J. Z. Zhao, R. Yu, H. M. Weng, and Z. Fang, Topological nodeline semimetal in compressed black phosphorus, Phys. Rev. B 94, 195104 (2016).

[8] P. L. Gong, D. Y. Liu, K. S. Yang, Z. J. Xiang, X. H. Chen, Z. Zeng, S. Q. Shen, and L. J. Zou, Hydrostatic pressure induced three-dimensional Dirac semimetal in black phosphorus, Phys. Rev. B 93, 195434 (2016).

[9] C. H. Li, Y. J. Long, L. X. Zhao, L. Shan, Z. A. Ren, J. Z. Zhao, H. M. Weng, X. Dai, Z. Fang, C. Ren, and G. F. Chen, Pressureinduced topological phase transitions and strongly anisotropic magnetoresistance in bulk black phosphorus, Phys. Rev. B 95, 125417 (2017).

[10] D.-K. Seo and R. Hoffmann, What determines the structures of the group 15 elements? J. Solid State Chem. 147, 26 (1999).
[11] Y. Akahama, M. Kobayashi, and H. Kawamura, Simple-cubicsimple-hexagonal transition in phosphorus under pressure, Phys. Rev. B 59, 8520 (1999).

[12] J. Wittig, B. Bireckoven, and T. Weidlich, in Solid State Physics Under Pressure, edited by S. Minomura (KTK Scientific, Tokyo, 1985), p. 217.

[13] H. Kawamura, I. Shirotani, and K. Tachikawa, Anomalous superconductivity in black phosphorus under high pressures, Solid State Commun. 49, 879 (1984).

[14] H. Kawamura, I. Shirotani, and K. Tachikawa, Anomalous superconductivity and pressure induced phase transitions in black phosphorus, Solid State Commun. 54, 775 (1985).

[15] M. Karuzawa, M. Ishizuka, and S. Endo, The pressure effect on the superconducting transition temperature of black phosphorus, J. Phys.: Condens. Matter 14, 10759 (2002).

[16] J. Guo, H. H. Wang, F. von Rohr, W. Yi, Y. Zhou, Z. Wang, S. Cai, S. Zhang, X. D. Li, Y. C. Li et al., The vital role of hole-carriers for superconductivity in pressurized black phosphorus, Phys. Rev. B 96, 224513 (2017).

[17] M. Rajagopalan, M. Alouani, and N. E. Christensen, Calculation of band structure and superconductivity in the simple cubic phase of phosphorus, J. Low Temp. Phys. 75, 1 (1989).

[18] M. Aoki, N. Suzuki, and K. Motizuki, Electronic band structure and superconductivity in simple-cubic phosphorus, J. Phys. Soc. Jpn. 56, 3253 (1987).

[19] H. Nagara, K. Mukose, T. Ishikawa, M. Geshi, and N. Suzuki, Origin of the simple modulated structures and the pressure induced superconductivity, J. Phys.: Conf. Series 215, 012107 (2010).

[20] L. W. Nixon, An investigation of the pressure-induced superconductivity transition temperature $T_{c}$ and related properties 
in elemental superconductors, Ph.D. thesis, George Mason University, 2010.

[21] K. T. Chan, B. D. Malone, and M. L. Cohen, Pressure dependence of superconductivity in simple cubic phosphorus, Phys. Rev. B 88, 064517 (2013).

[22] J. A. Flores-Livas, A. Sanna, A. P. Drozdov, L. Boeri, G. Profeta, M. Eremets, and S. Goedecker, Interplay between structure and superconductivity: Metastable phases of phosphorus under pressure, Phys. Rev. Mater. 1, 024802 (2017).

[23] P. B. Allen, Neutron spectroscopy of superconductors, Phys. Rev. B 6, 2577 (1972).

[24] A. Y. Liu, I. I. Mazin, and J. Kortus, Beyond Eliashberg Superconductivity in $\mathrm{MgB}_{2}$ : Anharmonicity, Two-Phonon Scattering, and Multiple Gaps, Phys. Rev. Lett. 87, 087005 (2001).

[25] W. L. McMillan, Transition temperature of strong-coupled superconductors, Phys. Rev. 167, 331 (1968).

[26] P. B. Allen and R. C. Dynes, Transition temperature of strongcoupled superconductors reanalyzed, Phys. Rev. B 12, 905 (1975).

[27] P. Morel and P. W. Anderson, Calculation of the superconducting state parameters with retarded electron-phonon interaction, Phys. Rev. 125, 1263 (1962).

[28] P. B. Allen and B. Mitrovic, Theory of superconducting $T_{c}$, Solid State Phys. 37, 1 (1983).

[29] A. Sanna, S. Pittalis, J. K. Dewhurst, M. Monni, S. Sharma, G. Ummarino, S. Massidda, and E. K. U. Gross, Phononic selfenergy effects and superconductivity in $\mathrm{CaC}_{6}$, Phys. Rev. B 85, 184514 (2012).

[30] P. E. Blöchl, Projector augmented-wave method, Phys. Rev. B 50, 17953 (1994).

[31] G. Kresse and J. Hafner, Ab initio molecular dynamics for liquid metals, Phys. Rev. B 47, 558 (1993).

[32] G. Kresse and J. Furthmüller, Efficiency of ab-initio total energy calculations for metals and semiconductors using a plane-wave basis set, Comput. Mater. Sci. 6, 15 (1996).

[33] G. Kresse and J. Furthmüller, Efficient iterative schemes for ab initio total-energy calculations using a plane-wave basis set, Phys. Rev. B 54, 11169 (1996).
[34] J. P. Perdew, K. Burke, and M. Ernzerhof, Generalized Gradient Approximation Made Simple, Phys. Rev. Lett. 77, 3865 (1996).

[35] O. K. Andersen, Linear methods in band theory, Phys. Rev. B 12, 3060 (1975).

[36] The ELK FP-LAPW code: http://elk.sourceforge.net/.

[37] J. K. Dewhurst, S. Sharma, C. Ambrosch-Draxl, and B. Johansson, First-principles calculation of superconductivity in holedoped LiBC: $T_{c}=65$ K, Phys. Rev. B 68, 020504(R) (2003).

[38] K. Koepernik and H. Eschrig, Full-potential nonorthogonal local-orbital minimum-basis band-structure scheme, Phys. Rev. B 59, 1743 (1999).

[39] F. Giustino, M. L. Cohen, and S. G. Louie, Electron-phonon interaction using Wannier functions, Phys. Rev. B 76, 165108 (2007).

[40] S. Poncé, E. R. Margine, C. Verdia, and F. Giustino, EPW: Electron-phonon coupling, transport and superconducting properties using maximally localized Wannier functions, Comput. Phys. Commun. 209, 116 (2016).

[41] P. Giannozzi et al., QUANTUM ESPRESSO: a modular and opensource software project for quantum simulations of materials, J. Phys.: Condens. Matter 21, 395502 (2009).

[42] S. M. Clark and J. M. Zaug, Compressibility of cubic white, orthorhombic black, rhombohedral black, and simple cubic black phosphorus, Phys. Rev. B 82, 134111 (2010).

[43] M. Monni, F. Bernardini, A. Sanna, G. Profeta, and S. Massidda, Origin of the critical temperature discontinuity in superconducting sulfur under high pressure, Phys. Rev. B 95, 064516 (2017).

[44] J. Ziman, Electrons and Phonons (Oxford University Press, Oxford, 1960).

[45] R. Akashi and R. Arita, Development of Density-Functional Theory for a Plasmon-Assisted Superconducting State: Application to Lithium Under High Pressures, Phys. Rev. Lett. 111, 057006 (2013)

[46] A. V. Krukau, O. A. Vydrov, A. F. Izmaylov, and G. E. Scuseria, Influence of the exchange screening parameter on the performance of screened hybrid functionals, J. Chem. Phys. 125, 224106 (2006) 